\title{
RESEARCH HIGHLIGHT Inosine: novel microbiota-derived immunostimulatory metabolite
}

\author{
Guido Kroemer (D) ${ }^{1,2,3,4,5}$ and Laurence Zitvogel ${ }^{4,6,7,8,9}$ \\ Cell Research (2020) 30:942-943; https://doi.org/10.1038/s41422-020-00417-1
}

In a recent paper published in Science, Mager et al. identify inosine as a metabolite produced by Bifidobacterium pseudolongum that improves the efficacy of anticancer immunotherapy in mice. Inosine produced in the gut reaches adenosine A2A receptors of T lymphocytes infiltrating tumors; thus, inosine joins an expanding list of immunostimulatory metabolites produced in the gut.

The immune system loses its capacity to control tumor growth in conditions of gut dysbiosis, a microbial imbalance characterized by a rarefied ecological diversity with reduction of beneficial commensals and, in some cases, an overabundance of potentially harmful species. Thus, the presence of specific bacterial species in the intestinal lumen is required for immunotherapy targeting CTLA-4 or PD-1 to reduce tumor growth, as this has been shown in preclinical experiments involving germ-free mice or specific pathogen-free mice sterilized with broad-spectrum antibiotics, followed by recolonization of the gut with defined microbial strains. ${ }^{1-4}$ Mechanistically, it appears that specific species in the microbiota can provide co-stimulatory signals acting on pattern recognition receptors to facilitate immune activation in the form of "co-stimuli", ${ }^{5}$ generate immunostimulatory metabolites (such a polyamines and vitamins $\mathrm{B} 3$ or $\mathrm{B} 6)^{6,7}$ or provide antigens that cross-react with tumor-associated antigens, thus boosting the recognition and destruction of malignant cells by cytotoxic $T$ lymphocytes. $^{8}$

In a recent study, Mager et al. ${ }^{9}$ set out to show that combined immune checkpoint blockade (ICB) targeting PD-1 or CTLA-4 (or monotherapy targeting CTLA-4) alone could reduce the incidence and the size of inflammation-induced colon cancers in mice, correlating with an enrichment of some bacterial species including Akkermansia muciniphila (A. muciniphila) and Bifidobacterium pseudolongum (B. pseudolongum). A. muciniphila has already been associated with improved immunotherapy outcome in mice and patients, ${ }^{2}$ prompting Mager et al. to investigate B. pseudolongum that turned to robustly enhance the immunotherapeutic effect of CTLA-4 or (less so) PD-1 blockade when supplemented orally to germ-free mice bearing transplantable MC38 colon cancers. ${ }^{9}$ This effect was lost upon heat inactivation of the bacterium. Although B. pseudolongum had no antitumor activity on its own (without ICB), it did increase the abundance of $\mathrm{CD}^{+}{ }^{+}$cells expressing the Th1 master transcription regulator T-bet in the gut-associated lymphoid tissue (GALT), as well as that of $\mathrm{CD}^{+}{ }^{+}$-bet $^{+}{ }^{+}$cells (Tc1 cells) in the ileum in the absence of CTLA-4 blockade. In combination with CTLA-4 blocking antibody, B. pseudolongum also enhanced Th1/Tc1 cell activation and effector function as evidenced by enhanced interferon- $\gamma$ (IFN- $\gamma$ ) production by splenic $\mathrm{CD}^{+}$and $\mathrm{CD}^{+} \mathrm{T}$ lymphocytes in tumor-free mice. Thus, $B$. pseudolongum induces Th1/TC1 differentiation and, together with anti-CTLA-4, activation of IFN- $\gamma$-producing Th1/Tc1 effector T cells. ${ }^{9}$

Searching for the mechanisms that account for the immunotherapy-enhancing effects of intestinal B. pseudolongum colonization, Mager et al. found that serum from mice treated with CTLA-4 blockade plus $B$. pseudolongum (but not from mice treated with either agent alone) was sufficient to reduce tumor growth and elicit strong antitumor immunity in germ-free mice. Metabolomic analyses revealed that inosine as well as its degradation factors xanthine and hypoxanthine were overabundant in sera from $B$. pseudolongum monocolonized mice as compared to germ-free controls. The local concentration of inosine was highest in the duodenum and gradually decreased along the small and large intestines. Thus, it appears that inosine produced by $B$. pseudolongum in the proximal small intestine accounts for its rise in systemic circulation. While inosine alone could not replace the effects of $B$. pseudolongum as an enhancer of tumor control by CTLA-4 blockade in the germ-free mice, the combination of inosine and $\mathrm{CpG}$ as a co-stimulus was efficient in doing so. This effect was not only observed in transplantable colon carcinomas, but also in mouse models of heterotopic bladder cancer and melanoma. ${ }^{9}$

In vitro assays revealed that inosine stimulates Th1 differentiation, specifically in the presence of costimulation with anti-CD3/ anti-CD28 antibodies or, alternatively supplementation with IFN- $\gamma$, but not in its absence, an effect that was abolished by an adenosine $A_{2 A}$ receptor $\left(A_{2 A} R\right)$ antagonist, but mimicked by cellpermeable cyclic AMP (db-cAMP), a signaling molecule downstream of $A_{2 A} R s$. More importantly, in mice bearing $T$ lymphocytes that lack $A_{2 A} R s$, the combination of CTLA-4 blockade and $B$. pseudolongum monocolonization and (or that of CTLA blockade plus inosine and $\mathrm{CpG}$ ) lost its tumor growth-reducing activity, confirming the idea that inosine must act on $A_{2 A} R s$ on $T$ cells. Importantly, the absence of $A_{2 A} R s$ on $T$ lymphocytes also compromised the antitumor activity of $A$. muciniphila (producing inosine) and Lactobacillus johnsonii (producing hypoxanthine, which also acts on $A_{2 A} R s$ ). Inosine (without $C p G$ ) was able to improve the efficacy of anti-CTLA-4 therapy in the presence of

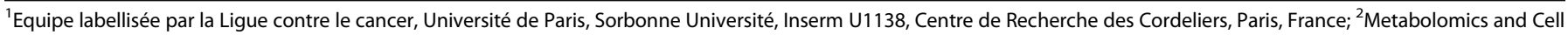

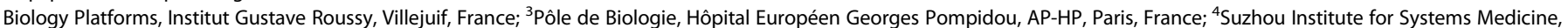

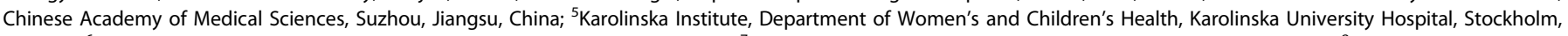

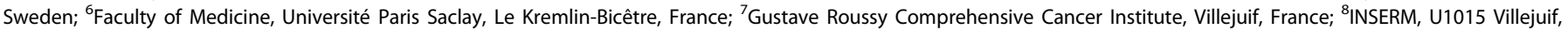
France and ${ }^{9}$ Center of Clinical Investigations in Biotherapies of Cancer (CICBT), Villejuif 1428, France

Correspondence: Guido Kroemer (Kroemer@orange.fr)

Published online: 21 September 2020 


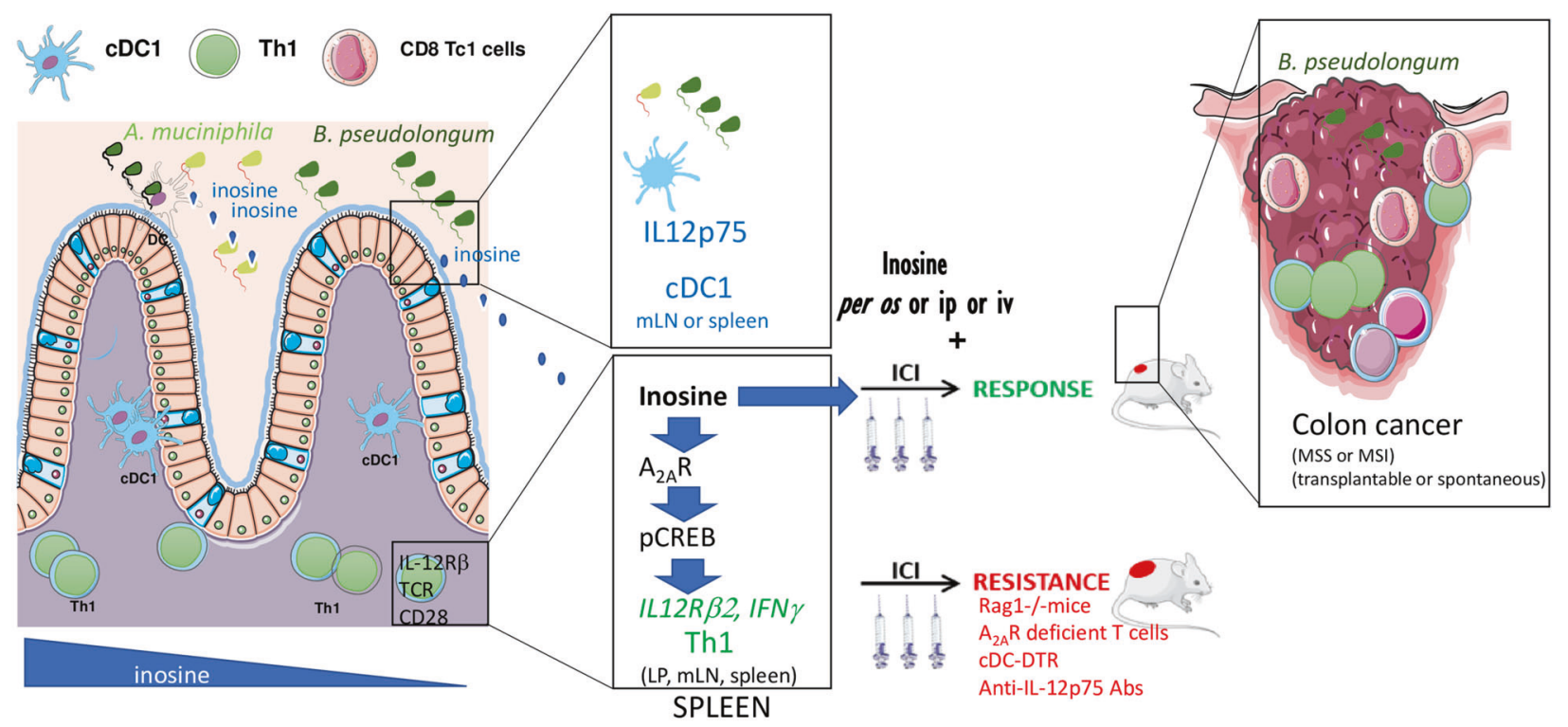

Fig. 1 Inosine/adenosine receptor A2-dependent co-stimulation of pro-Th1 and pro-Tc1 cells is required for the immunostimulatory effect of gut-resident $B$. pseudolongum in colon cancer immunotherapy. During immunotherapy with the immune checkpoint inhibitor (ICI) anti-CTLA-4 (combined with either CpG or anti-PDL-1 antibody), inosine produced by gut-resident microbial species (such as B. pseudolongum and A. muciniphila) in a descending gradient from the duodenum to the ileum leads to $A_{2 A} R$-dependent costimulatory effects on $T$ cells, Th1/ Tc1 differentiation and accumulation in tumor beds (DSS-AOM colon cancer, subcutaneous MC38 colon cancer or Msh2LoxP/LoxPVillin-Cre model). Indeed, $A_{2 A} R$ signaling in $\mathrm{T}$ lymphocytes is mandatory for the immunotherapy-promoting effects of $B$. pseudolongum and allows for upregulation of IL12Rß2 and IFNy transcription, while IL-12 is secreted by CDC1 in contact with bacteria including $B$. pseudolongum, likely in secondary lymphoid organs. $\mathrm{PCREB}$, phosphorylation of $C A M P$ response element-binding protein; $\mathrm{mLN}$, mesenteric lymph node; $L P$, lamina propria; MSI, microsatellite instable; MSS, microsatellite stable; ip, intraperitoneally; iv, intravenously; DTR, diphteria toxin receptor; per os, orally.

complex microbiota, for instance in specific pathogen-free mice, as well as in gnotobiotic mice stably colonized with a defined microbiota consisting of 12 bacterial species that do not include $B$. pseudolongum. Additional experimentation confirmed the capacity of $B$. pseudolongum-containing bacterial consortia to improve immunotherapy outcome in a mouse model of mismatch repair deficient (MMRD) small intestinal cancer (driven by the deletion of Msh2 in enterocytes) or mismatch repair proficient (MMRP) colon cancers (driven by the deletion of APC and the activation of RAS in colonocytes), as well as the need for IL-12-producing Type-1 dendritic cells (cDC1) for such effects.'

Altogether (Fig. 1), these results suggest that B. pseudolongum and other bacterial species (such as $A$. muciniphila) can produce inosine that has systemic effects, distant from the gut, on $A_{2 A} R s$ expressed by $T$ lymphocytes. In addition, $B$. pseudolongum and other microbes can elicit IL-12 production by CDC1 cells, through the production of molecular entities that remain to be chemically defined but that can be advantageously replaced by CpG. In combination, these signals suffice to boost the efficacy of ICBbased anticancer immunotherapy. The fact that $B$. pseudolongum still improves immunotherapy in specific pathogen-free mice or gnotobiotic mice ${ }^{9}$ strongly suggests that distinct bacteria use different mechanisms to stimulate immunosurveillance.
This work supports the previously reported contention ${ }^{10}$ that the small intestine microbiota dictates the prognosis of tumors located in the large intestine, offering potential solution to the resistance of microsatellite stable colon cancers harboring T-cell infiltrates to the combination of oxaliplatinum and PD-1-based therapies, by supplementing patients with oral inosine-producing probiotics or systemic inosine.

\section{ADDITIONAL INFORMATION}

Competing interests: G.K. and L.Z. are scientific co-founders of everlmmune.

\section{REFERENCES}

1. Vetizou, M. et al. Science 350, 1079-1084 (2015).

2. Routy, B. et al. Science 359, 91-97 (2018).

3. Matson, V. et al. Science 359, 104-108 (2018).

4. Li, Y. et al. Cell Rep. 30, 1753-1766 (2020).

5. Zitvogel, L. et al. Science 359, 1366-1370 (2018).

6. Rooks, M. G. et al. Nat. Rev. Immunol. 16, 341-352 (2016).

7. Madeo, F. et al. Science 359, eaan2788 (2018).

8. Fluckiger, A. et al. Science 369, 936-942 (2020).

9. Mager, L. F. et al. Science https://doi.org/10.1126/science.abc3421 (2020).

10. Roberti, P. M. et al. Nat. Med. 26, 919-931 (2020). 